\title{
¿Puedo conducir un automóvil, doctor? Evaluación para el manejo de automóviles en personas con discapacidad y su importancia en la rehabilitación
}

\author{
DANIELA GARCÍA P. ${ }^{1,2}$
}

\author{
Can I drive a car, doctor? Car driving \\ evaluation for people with disability and \\ the importance in rehabilitation
}

One of the main objectives in the rehabilitation of people with disability is to give them the possibility of mobilizing independently and a car is a modern and effective tool for achieving this objective. It is essential to make a specific assessment that includes at least the visual, cognitive and motor area before determining whether the individual can drive a car, or also what kind of adaptations may be required. It is also essential to properly know the Traffic Law in force in our country to be able to guide the steps that the patient has to follow to obtain a driver's license. The objective of this review is to study deeply this interesting subject and all the edges that have been mentioned above.

(Rev Med Chile 2010; 138: 243-250).

Key words: Automobile driver examination; Disability evaluation; Quality of life.

4 n el año 2004, el Fondo Nacional de Discapacidad (FONADIS) realizó el primer estudio formal para evaluar la discapacidad en Chile ${ }^{1}$. Dentro de sus resultados destacó que 2.068.072 chilenos (equivalentes 12,9\% de la población) padecen y deben vivir con algún tipo de discapacidad y esto afecta a personas de todos los niveles socioeconómicos. A pesar de que la mayoría de ellos se cataloga dentro del grado leve de discapacidad y se encuentra en el rango etario de 30 a 64 años, más del 70\% no realiza un trabajo remunerado ni participa de alguna organización social, de cualquier tipo. Los encuestados señalaron la dificultad para desplazarse como una de las principales causas que explican esto último.

Se puede desprender que la capacidad para movilizarse es primordial y fundamental para alcanzar la independencia al portar algún tipo de discapacidad, lo que a su vez permitirá mejorar la calidad de vida y la autoestima, factores de gran importancia en el proceso de rehabilitación. Para que la persona pueda recuperar su función social y laboral es necesario que sea capaz de abandonar la protección del hogar y reinsertarse en la comunidad.

A pesar de los grandes avances que se han realizado en nuestro país en el último tiempo, todavía existen grandes restricciones para que las personas con alguna limitación física o mental puedan utilizar el transporte público, por lo que poder conducir un automóvil se convierte en una alternativa muy importante para la rehabilitación integral.

La destreza para conducir un automóvil es una eficaz herramienta en la sociedad contemporánea, no sólo para conseguir y mantener la independencia de la persona, sino que también es símbolo de adultez, ayuda a mantener la autoestima y es un 
paso importante en la exploración vocacional. Incluso hay estudios que han demostrado que los pacientes discapacitados que no logran conducir un automóvil presentan una mayor incidencia de episodios depresivos comparados con aquellos que sí conducen ${ }^{2}$. Este hecho es aun más acentuado en aquellos individuos que conducían antes de sufrir la discapacidad, porque notan que el dejar de hacerlo significa un cambio dramático en sus vidas.

La capacidad de movilizarse en forma independiente favorece el crecimiento de múltiples áreas, tales como el desempeño laboral, una vida social activa, mantener las amistades, participar de las actividades familiares, poder realizar las compras personales y del hogar, planear las vacaciones, realizar paseos, reaccionar frente a una urgencia, mantener los horarios personales, etc.

Pero hay que considerar que conducir un automóvil es una actividad muy compleja que requiere de la indemnidad e integración de múltiples habilidades 3 . Dentro de las más importantes se encuentran una buena visión y motricidad gruesa y fina, habilidades cognitivas complejas, rápida velocidad de reacción frente a estímulos y la capacidad de atender y procesar más de un estímulo a la vez. La Tabla 1 resume las funciones ejecutivas, sensoriales y motoras involucradas en el manejo óptimo de un vehículo motorizado.

\section{Tabla 1. Resumen de las diversas funciones ejecutivas necesarias para lograr una conducción vehicular segura}

Habilidades involucradas en la conducción de
un automóvil
Movilidad
Visión
Audición
Habilidades cognitivas
Pensamiento abstracto
Integración de señales
Velocidad de reacción
Estabilidad emocional
Buena tolerancia física y mental
Rápida velocidad de procesamiento de información
Memoria
Atención

Existe un temor global, infundado, de que las personas con discapacidad que conducen un vehículo motorizado pueden acarrear un peligro para ellos mismos y para quienes los rodean. Sin embargo, la mayoría de los estudios realizados sugiere lo contrario y muestra que esta población tiene una tasa de accidentes menor que el resto de los conductores ${ }^{4}$. Lo primordial es realizar una buena evaluación para detectar las dificultades que puede presentar cada paciente.

La tecnología actual facilita que cada vez más personas que padecen de alguna enfermedad discapacitante puedan aspirar a conducir un automóvil. El paciente, en busca de apoyo, información, consejo y orientación, transmite sus inquietudes a su médico tratante. Es deber del profesional auxiliar a estos pacientes y derivarlo oportunamente a un médico especialista en Medicina Física y Rehabilitación o Fisiatría para ser sometido a una evaluación completa, entrenamiento, e indicar la prescripción básica de las adaptaciones requeridas en un vehículo, para cada paciente en particular.

El objetivo de este trabajo es revisar la literatura existente respecto a este tema, incluyendo la evaluación, manejo, y marco legal que permite que las personas con discapacidad puedan aspirar a conducir un automóvil.

\section{Evaluación del paciente discapacitado}

\section{a) Conceptos generales}

Lo esencial para determinar si una persona tiene o no las capacidades físicas y psíquicas para manejar un vehículo, es no olvidar que cada individuo es distinto y que no es el tipo de patología lo que limita la decisión a tomar. Al enfrentarnos ante esta interrogante hay que considerar primero las implicancias éticas y legales pues, como ya se mencionó, son múltiples las áreas que se ven afectadas tanto si se le entrega como o si se le niega a una persona la licencia para conducir. Tampoco hay que olvidar que como médicos debemos respetar el derecho de confiabilidad del paciente, pero no poner en riesgo ni a él ni a su entorno $y$, por sobretodo, respetar la Ley de Tránsito vigente en nuestro país ${ }^{5}$. Otros puntos importantes que no se deben olvidar, son la consignación de todos los medicamentos que está utilizando la persona y tener claros los efectos que estos pueden acarrear sobre la calidad de la conducción. También 
es importante la opinión de la familia, pues ellos serán los que observarán al paciente en el día a día y pueden detectar problemas previamente no identificados ${ }^{6}$. Por último, es también importante mencionar que se ha demostrado que algunas de las características previas a la discapacidad del paciente también pueden influir en los resultados de sus evaluaciones. A modo de ejemplo podemos mencionar su historia de conductor previo, considerando sobre todo la cantidad de accidentes, infracciones y su perfil como conductor; el uso de drogas o alcohol y el tipo de personalidad previa, con énfasis en la detección de componentes de agresividad e impulsividad ${ }^{7}$.

Para seguir un orden lógico y registrar todos los datos de importancia, Thomas y Hughes ${ }^{8}$ crearon recientemente una nemotecnia bastante práctica denominada LEAN (Licence-Eligibility - Ability Notification). El primer paso consiste en averiguar si el paciente ha tenido alguna vez alguna licencia de conducir, de qué tipo, y si está vigente o no. Luego hay que determinar si el paciente en su condición actual tiene alguna posibilidad de conducir, según lo que se estipula en la Ley local. La habilidad del paciente debe ser estimada utilizando los tipos de evaluaciones que más se ajusten a la persona $y$, por último, notificar los resultados al paciente y a las entidades que correspondan en cada país.

\section{b) Evaluación teórica}

Existe una gran diversidad de evaluaciones que se pueden utilizar para determinar las facultades para manejar que presenta un individuo con discapacidad. Diversos estudios han demostrado la utilidad de pruebas específicas para esto, así como también la incorporación de pruebas más generales que han sido acreditadas como eficientes en la delimitación de los problemas al conducir, en estos pacientes. Se considera también la combinación de distintas baterías de exámenes cuya sumatoria potencia la validez de los resultados ${ }^{9,10}$. La evaluación inicial debe incluir al menos un examen completo de la capacidad visual y la medición de las capacidades perceptuales, motoras y cognitivas ${ }^{11}$.

Noventa a 95\% de los estímulos que se reciben al manejar un vehículo son de tipo visual, por lo que se debe incluir una apreciación muy completa de esta área ${ }^{12}$. La valoración debe incluir al menos la agudeza visual a través del test de Snellen, los campos visuales por medio de confrontación, la sensibilidad de contraste a colores y la acomo- dación de la vista a cambios en la iluminación ${ }^{12}$. Dentro del área cognitiva, la evaluación mínima debe incluir la capacidad de memoria, la percepción visual, el procesamiento visual, las habilidades visioespaciales, la atención selectiva y dividida, y las habilidades ejecutivas ${ }^{11}$. En la evaluación motora hay que consignar la fuerza motora, utilizando la Escala de Fuerza Muscular del Medical Research Council (MRC) ${ }^{13}$, los rangos osteomusculares de las extremidades y la sensibilidad táctil y propioceptiva. Lo ideal es siempre comenzar con evaluaciones teóricas en la consulta, antes de comenzar las pruebas prácticas, pues son de menor costo, son menos intimidantes para el paciente, todo se hace dentro de un marco de mayor seguridad $y$, por sobre todo, permiten evaluar habilidades específicas en forma separada ${ }^{14}$.

Son múltiples las pruebas teóricas que se han utilizado con el fin de extrapolar la seguridad que presentará el paciente al conducir. La Tabla 2 muestra las más utilizadas y analizaremos algunas de ellas en mayor profundidad.

Tabla 2. Lista de las múltiples pruebas que se han utilizado para evaluar las habilidades requeridas para conducir en forma segura un automóvil

\section{Evaluaciones que se pueden utilizar para de- terminar las capacidades de conducir de cada paciente}

Trail Making Test

Visual Form Recognition Test

Single and Double Letter Cancellation

Cognitive Behavioral Driver's Inventory

Dynavision Assessment Battery

Bells Test

Motor-Free Visual Perception Test

Useful Field of Vision

Complex Reaction Timer

Money Road Map of Direction Test

Charron Test

Prueba del Reloj

Prueba de Rey

(Adaptada de Arakelian M. Prediction of return to driving post-CVA: an evidence-based review. Evidence-Based Symposium: December 2003, School of Occupational Therapy, University of Puget Sound). 
Muchas evaluaciones que no fueron creadas en forma específica para evaluar las capacidades frente al volante, han demostrado utilidad. Dentro de esta categoría está el Test de Cancelación de Letras (Single and Double Letter Cancellation) que se utiliza para detectar y medir la severidad del déficit en la exploración visual y atención visual ${ }^{15}$, y el Motor-Free Visual Perception Test (MFVPT) que permite medir la habilidad de percepción visual a través de alternativas visuales, excluyendo el uso de funciones motoras gruesas9. También se ha utilizado el Trail Making Test que se categoriza como una prueba de tipo cognitiva y ayuda a medir fallas de atención y cálculo. Este último, cuando se combina con el MFVPT, ha demostrado una buena correlación con el nivel de desempeño al conducir un automóvil en los pacientes con un accidente cerebrovascular $(\mathrm{ACV})^{16}$. Otro ejemplo es el Useful Field of View Test (UFOV), que ayuda a determinar el área del campo visual en la que el paciente puede procesar rápidamente y reaccionar frente a una información visual y detecta problemas en la función visual, velocidad de procesamiento visual y habilidades de atención visual ${ }^{17}$.

También se han elaborado pruebas especialmente para determinar las habilidades que una persona presenta para conducir un vehículo. Entre ellas se encuentra el Money Road-Map Test (MRMT) que se basa en un mapa de una ciudad con una ruta determinada a seguir y ayuda a medir la orientación izquierda/derecha, con y sin rotación mental egocéntrica en el espacio ${ }^{18}$. Otra evaluación que ha sido utilizada ampliamente, desde su creación en 1988, por la completa información que entrega, es el Cognitive Behavioral Driver's Inventory (CBDI) que consiste en una serie de tareas que requieren de la integración de múltiples áreas como la atención, concentración, tiempo de reacción, toma rápida de decisiones, exploración visual, alerta visual, atención dividida, discriminación de estímulos, coordinación visiomotora y secuencia visual, entre otros ${ }^{19}$. Por último, mencionaremos el Larson Driver's Stress Profile (LDSP), que está compuesto por un cuestionario que intenta detectar problemas conductuales que pueden interferir en el manejo del automóvil, centrado en el componente de agresividad e impulsividad $^{20}$.

\section{c) Evaluación práctica}

Una vez que se han realizado todas las evalua- ciones motoras, cognitivas y conductuales que se estimen convenientes para cada caso, es necesario pasar a una evaluación práctica. Hay lugares que cuentan con un paso previo que consiste en los simuladores de manejo, que han mostrado ser útiles. Los simuladores consisten fundamentalmente en un aparato que simula el interior de un vehículo, pero en el lugar del parabrisas tiene una pantalla de video que proyecta una ruta que debe seguir el individuo evaluado. La utilidad de este tipo de pruebas consiste en que se puede evaluar el desempeño real del paciente tras el volante y la integración de todas las habilidades requeridas para la conducción de un vehículo, sin el riesgo físico ni de estrés que conlleva una prueba práctica en sí. Los institutos que los utilizan han reportado resultados confiables y han evidenciado una buena correlación entre los resultados de este tipo de pruebas y la evaluación neuropsicológica formal que se les realiza a estos pacientes para detectar dificultades al conducir ${ }^{21}$. La tecnología actual ha permitido que se pueda avanzar aun más y hay investigadores que han incorporado el sistema de realidad virtual en los simuladores de manejo para la evaluación de pacientes con traumatismo encéfalo craneano $(\mathrm{TEC})^{22}$. Los resultados son prometedores, pero faltan más estudios al respecto.

A pesar de las múltiples evaluaciones que han demostrado utilidad para apreciar las habilidades para conducir de las personas con discapacidad, ninguna de ellas puede reemplazar la evaluación práctica. Colocar al paciente tras el volante y lograr que pueda dar una vuelta en el automóvil sigue siendo uno de los pilares más sólidos en la resolución final ${ }^{23}$. Es primordial siempre tener en cuenta que no basta con que la persona sea capaz de girar el volante, frenar y acelerar, sino que conducir un vehículo conlleva muchos pasos más, que van desde poder abrir la puerta del auto hasta poder descender de él (Tabla 3 ).

Por último, es importante recalcar que para que el paciente pueda conducir sin problemas, no basta con que pueda conducir un auto adaptado sino que, además, deben existir facilidades para que pueda utilizarlo. La American Medical Association (AMA) ha desarrollado las características básicas que debe tener el transporte para ser amistoso para la persona con limitaciones físicas: disponibilidad, accesibilidad, aceptación de los demás, adaptación y la disponibilidad de recursos económicos ${ }^{24}$. 


\section{Tabla 3. Lista de acciones que deben ser evaluadas por el examinador al momento de realizar una evaluación práctica de manejo en una persona con discapacidad}

Acciones que se deben evaluar durante la prue-
ba práctica
Chapa del auto
Abrir la puerta
Acomodarse en el asiento del piloto
Ajustar el cinturón de seguridad
Colocar la llave en el contacto
Encender el auto
Acomodar el espejo retrovisor
Acomodar los espejos laterales
Sacar el freno de mano
Usar el embrague
Girar el manubrio
Acelerar
Frenar
Pasar los cambios
Señalizar
Prender las luces
Usar el limpiaparabrisas
Uso de otras funciones: Radio, calefacción, aire acondi-
Cionado y subir ventanas
Cerrar el auto
Barir y cerrar la maleta
Auto

\section{Aspectos legales en Chile}

En Chile existen leyes para hacer más fácil la movilización de la persona discapacitada y así favorecer su integración social, pero, lamentablemente, existe muy poca fiscalización para objetivar su cumplimiento y prácticamente no existe penalización frente a una infracción a ellas. Por otra parte, hay escasa consideración, respeto y empatía del resto de los conductores hacia los conductores discapacitados, lo que se ve en claro manifiesto con la utilización inapropiada que existe de los estacionamientos preferenciales.

Para orientar, guiar y apoyar a una persona discapacitada en forma íntegra cuando presenta interés por manejar un automóvil, es trascendental conocer a fondo la Ley actualmente vigente para poder atenerse a ella. En Estados Unidos de Norteamérica y algunos países de Europa, como Inglaterra, se ha avanzado mucho en el último tiempo y se han creado entidades encargadas de clarificar las leyes para poder clasificar en forma eficiente a los pacientes y dejar menos cabida a la interpretación personal ${ }^{8,25-27}$.

En la realidad chilena hay que conocer la definición de discapacidad que se incluye en el Artículo $\mathrm{N}^{\circ} 3$ de la Ley de Integración Social para Personas con Discapacidad ${ }^{28}$ para tener claro cuáles pacientes se encuadran en ella. Basándose en el cumplimento de lo pactado en la Ley de Integración, se han tomado múltiples medidas cuyo fin es facilitar la vida a los individuos con discapacidad. Algunas de estas disposiciones son el acceso fácil y uso preferencial del transporte público, la facilidad para importar vehículos adaptados, los estacionamientos preferenciales, y la reducción de $25 \%$ en el monto a pagar por el permiso de circulación, entre otras.

Es necesario analizar con más profundidad lo que concierne a la importación de vehículos adaptados, ya que es una consulta recurrente de las personas con discapacidad, por su alto interés en conducir un automóvil. La Ley define en forma concisa cuáles son los individuos que pueden optar a este beneficio ${ }^{29}$ :

"Las personas lisiadas a que se refiere el inciso anterior son aquellas que presentan incapacidad permanente para la marcha normal en virtud de lesiones orgánicas of funcionales que afectan uno o los dos miembros inferiores $y$, además, aquellas que conjuntamente a su incapacidad permanente para la marcha normal, sufran de la incapacidad absoluta de uno de los miembros superiores."

Esta definición fue hecha en el año 1969, por lo que su terminología está obsoleta y su aplicación actual no es fácil. Por ende, muchos pacientes con discapacidad que podrían beneficiarse de esta ayuda, quedan fuera de esta descripción. Además, la Ley estipula que el vehículo importado podrá ser utilizado sólo para movilizarse al trabajo, lugar de estudio, o a su rehabilitación. También hay un tope monetario de US\$20.974,64 para el valor del auto que será importado ${ }^{29}$. El beneficio de la importación de vehículos consiste en la rebaja de $50 \%$ del total de los derechos de aduana que 
corresponde pagar por la importación de un vehículo $^{29}$. Pero hay que consideran que este pago no existe cuando el auto se importa de un país con el que Chile tiene tratado de libre comercio, como, por ejemplo, Estados Unidos de Norteamérica. En estos casos no se obtendrá ningún beneficio adicional al importar un vehículo.

Si se considera lo pactado en la Ley de Tránsito concerniente a la obtención de licencias para conducir, en personas con discapacidad, se puede observar que varios artículos se refieren a este tema ${ }^{30}$. Entre ellos, destaca el derecho a obtener cualquier tipo de licencia, desde la A hasta la F, pero con restricción $\mathrm{N}^{\circ} 4$ que implica poder manejar sólo el vehículo adaptado. En el caso de la licencia de conducir clase B, que es la más frecuentemente solicitada por estos pacientes, la idoneidad física y psíquica del postulante debe ser acreditada por el Jefe del Gabinete Técnico del Departamento de Tránsito y Transporte Público Municipal y por el médico del mismo. Si no logra garantizarse la integridad física y psíquica, pero estas pueden alcanzarse con adaptaciones del vehículo, se le puede conceder la licencia, a criterio del examinador. Por otra parte, el Reglamento para el Otorgamiento de Licencias de Conducir en su Artículo $\mathrm{N}^{\circ} 3$ incluye una lista de alteraciones que, al estar presentes en un paciente, harán que éste sea calificado como "carente de aptitudes para manejar"31 (Tabla 4). Sin embargo, si el individuo logra pasar las pruebas señaladas por la Ley para obtener una licencia y lleva un certificado de su médico tratante que consigne que la patología está bien controlada, podría obtener una licencia de conducir, a criterio del examinador.

De todo lo anterior se puede deducir que la Ley chilena no es clara en lo que respecta al derecho de las personas con discapacidad para conducir un automóvil, por lo que el consejo y apoyo del médico especialista cobran vital importancia. Actualmente, se encuentra en el Congreso una nueva Ley que pretende modificar muchas de las falencias antes expuestas.

\section{Conclusiones}

En el mundo moderno, el automóvil es una pieza clave en la funcionalidad, integración social y laboral de personas con discapacidad. Es deber del médico tratante abordar este tema frente a una persona discapacitada. Si el paciente muestra inte-

\section{Tabla 4. Lista de personas consideradas como carentes de aptitudes para conducir, según el Reglamento Chileno para el Otorgamiento de Licencias de conducir}

\section{Personas carentes de aptitudes para manejar según el reglamento chileno}

1. Enfermedades con crisis de compromiso de conciencia.

2. Enfermedades que produzcan incapacidad para efectuar movimientos voluntarios rápidos.

3. Enfermedades con movimientos involuntarios.

4. Defecto anatómico o funcional que aún corregido le imposibilite el control del automóvil.

5. Capacidad ventilatoria funcional $\leq 40 \%$

6. Insuficiencia cardíaca grados III y IV

7. Insuficiencia coronaria crónica con capacidad de esfuerzo $<6$ METS.

8. Hipertensión arterial maligna.

9. Cardiopatía congénita con insuficiencia cardíaca o respiratoria.

10. Prótesis valvulares cardíacas, mitral o aórtica.

11. Diabetes mellitus tipo 1.

12. Insuficiencia renal crónica grado IV.

13. Diplopia no corregida.

14. Toxicomanías (drogas, alcohol o ambos) sin tratamiento.

15. Uso de sustancias que produzcan compromiso de conciencia, mala percepción, baja habilidad motriz, inestabilidad emocional y del juicio.

16. Aquel que no aprueba los exámenes sensométricos ni psicométricos.

Extraído de http://www.leychile.cl Navegar?idNorma=10426

rés por conducir un automóvil, debe ser derivado al Fisiatra para que sea evaluado y orientado en los pasos que debe seguir para obtener una licencia de conducir.

Agradecimientos: A los Abogados José Miguel Nalda M. y Carmen Palomer R., por su asesoría profesional y al Departamento Jurídico de FONADIS, por su orientación y consejo en cuanto al marco legal actualmente vigente en Chile. También agradezco al Sr. Dan Basore, terapeuta especialista en conducción de automóvil del Hospital MossRehab de Filadelfia, por su ayuda en la obtención de importante material bibliográfico. 


\section{Referencias}

1. Zondek A, Zepeda M, Recabarren E, González F, Mires L, Arancibia F, Aguirre E. Primer Estudio Nacional de la Discapacidad en Chile. Fondo Nacional de Discapacidad, Gobierno de Chile, 2004. Extraído de http://www. fonadis.cl $/$ index.php? seccion $=9 \&$ parametro $=79 \#$ centro, [Consultado el 3 de junio de 2009].

2. Legh-Smith J, Wade D, Langton R. Driving after a stroke. J Royal Soc Medicine 1986; 79: 200-8.

3. Akinwuntan A, Feys H, DeWeerdt W, Baten G, Kiekens C. Prediction of driving after a stroke: a prospective study. Neurorehabil Neural Repair 2006; 20: 417-23.

4. Akinwuntan A, Feys H, DeWeerdt W, Pauwels J, Baten G, Strypstein E. Determinants of driving after stroke. Arch Phys Med Rehabil 2002; 83: 334-41.

5. Wheatley C, Stefano M. Individualized assessment of driving fitness for older individuals with health disability, and age-related concerns. Traffic Injury Prevention 2008; 9: 320-7.

6. Hopewell A. Driving assessment issues for practicing clinicians. J Head Trauma Rehabil 2002; 17: 48-61.

7. Akinwutan A, Devos H, Feys H, Verheyden G, Baten G, Kiekens C, DeWeerdt W. Confirmation of the accurancy of a short battery to predict fitness-to-drive of stroke survivors without severe deficits. J Rehabil Med 2007; 39: 698-702.

8. Thomas R, Hughes T. Can I drive, doctor? LEAN thinking may help us answer the question. Pract Neurol 2009; 9: 71-9.

9. Bouillon L, Mazer B, Gelinas I. Validity of the cognitive behavioral driver's inventory in predicting driving outcome. America J Occupational Therapy 2006; 60: 420-7.

10. Szlyk J, Myers L, Zhang Y, Wetzel L, Shapiro R. Development and assessment of a neuropsychological battery to aid in predicting driving performance. J Rehabil Research Develop 2002; 39: 483-96.

11. Wang C, Kosinski C, Schwartzberg J. Physician's Guide to Assesing and Counseling Older Drivers, Chapter 3. American Medical Association, 2003. Extraído de http:// www.ama-assn.org/ama/pub/physician-resources/public-health/promoting-healthy-lifestyles/geriatric-health/ older-driver-safety/assessing-counseling-older-drivers. shtml, [Consultado el 9 de junio de 2009].

12. Wheatley C. Visual perceptual aspects of driving. Physical Disabilities 2001; 24: 1-4.

13. John J. Grading of muscle power: comparison of MRC and analogue scales by physiotherapists. Medical Research Counsil. Int J Rehabil Res 1984; 7: 173-81.

14. Arakelian M. Prediction of return to driving post-CVA: an evidence-based review. Evidence-Based Symposium:
December 2003, School of Occupational Therapy, University of Puget Sound. Extraído de http://www2.ups. edu/ot/evidence/2003/Arakelian.pps, [Consultado el 9 de junio de 2009].

15. Mazer B, Sofer S, Korner-Bitenky N, Gelinas I, Hanley J, Wood-Dauphinne S. Effectiveness of a visual attention retraining program on the driving performance of clients with stroke. Arch Phys Med Rehabil 2003; 84: 541-50.

16. Mazer B, Korner-Bitensky N, Sofer S. Prediciting ability to drive after stroke. Arch Phys Med Rehabil 1998; 79: 743-50.

17. Edwards J, Ross L, Wadley V, Clay O, Crowe M, Roenker $\mathrm{D}$, Ball K. The useful field of view test: normative data for older adults. Arch Clin Neuropsychol 2006; 21: 27586.

18. Vingerhoets G, Lanoo E, Bauwens S. Analysis of the money road-map test performance in normal and braindamaged subjects. Arch Clin Neuropsychol 1996; 11: $1-9$.

19. Engum E, Pedergrass T, Cron L, Lambert W. Cognitive behavioral driver's inventory. Cognitive Rehabil 1988; 6: 34-49.

20. Blanchard E, Barton K, Malta L. Psychometric properties of a measure of aggressive driving: the Larson driver'stress profile. Psychol Rep 2000; 87 (3 Pt 1): 88192.

21. Carroz A, Comte P, Nicolo D, Dériaz O, Vuadens P. Relevance of a driving simulator in the assessment of handicapped individuals. Ann Readap Med Phys 2008; 51: 358-65.

22. Lengerfelder J, Schultheis M, Al-Shihabi T, Mourant R, DeLuca J. Divided attention and driving: a pilot study using virtual reality technology. J Head Trauma Rehabil 2002; 17: 26-37.

23. Wheatley C, Di Stefano M. Individualized assessment of driving fitness for older individuals with health, disability, and age-related concerns. Traffic Injury Prev 2008; 9: 320-7.

24. Wang C, Kosinski C, Schwartzberg J. Physician's Guide to Assesing and Counseling Older Drivers, Chapter 9. American Medical Association, 2003. Extraído de http:// www.ama-assn.org/ama/pub/physician-resources/public-health/promoting-healthy-lifestyles/geriatric-health/ older-driver-safety/assessing-counseling-older-drivers. shtml, [Consultado el 4 de julio de 2009].

25. Brooks N, Hawley C. Return to driving after traumatic brain injury: a British perspective. Brain Injury 2005; 19: 165-75.

26. Tamietto M, Torrini G, Adenzato M, Pietrapiana P, Rago R, Perino C. To drive or not to drive (alter TBI)? 
A review of the literature and its implications for rehabilitation and future research. NeuroRehabil 2006; 21 : 81-92.

27. Wang C, Kosinski C, Schwartzberg J. Physician's Guide to Assesing and Counseling Older Drivers, Chapter 8. American Medical Association, 2003. Extraído de http:// www.ama-assn.org/ama/pub/physician-resources/public-health/promoting-healthy-lifestyles/geriatric-health/ older-driver-safety/assessing-counseling-older-drivers. shtml, [Consultado el 4 de julio de 2009].

28. Ley 19.284, promulgada el 14 de enero de 1994 por el Ministerio de Planificación y Cooperación. Extraído de http://www.fonadis.cl/transparencia/normativo/
LEY\%2019284.pdf, [Consultado el 4 de julio de 2009]. 29. Ley 17.238, promulgada el 13 de noviembre de 1969 por el Ministerio de Hacienda. Extraído de http://www. leychile.cl/Navegar?idNorma=28852 [Consultado el 4 de julio de 2009].

30. Ley de Tránsito: 18.290, Artículos $\mathrm{N}^{\circ} 13,14,19,20$ y 21. Extraído de http://www.servicioweb.cl/juridico/Ley\%20 del\%20transito.htm, [Consultado el 4 de julio de 2009].

31. Decreto $\mathrm{N}^{\circ} 170$ de la Ley del Tránsito, Artículo Nº3. Promulgado el 12 de diciembre de 1985 por el Ministerio de Transporte y Telecomunicaciones. Extraído de http:// www.leychile.cl/Navegar?idNorma=10426, [Consultado el 4 de julio de 2009]. 